\title{
Establishment of A Risk-Predicting Twenty-Two- Gene Signature Based on the Prognosis-Related RNA Binding Proteins of Breast Cancer
}

\section{Tong Zhu}

Shengjing Hospital of China Medical University

\section{Xudong Zhu}

Shengjing Hospital of China Medical University

Jiawen Bu

Shengjing Hospital of China Medical University

Yi Jiang

Shengjing Hospital of China Medical University

Jinqi Xue

Shengjing Hospital of China Medical University

Nan Niu

Shengjing Hospital of China Medical University

Xi Gu ( $\nabla$ gux1@si-hospital.org )

Shengjing Hospital of China Medical University https://orcid.org/0000-0002-4172-9821

Primary research

Keywords: Breast cancer, RBPs, Predictive signature, Prognosis

Posted Date: November 23rd, 2021

DOI: https://doi.org/10.21203/rs.3.rs-1065407/v1

License: (c) (i) This work is licensed under a Creative Commons Attribution 4.0 International License. Read Full License 


\section{Abstract \\ Background}

Breast cancer has been the leading cause of death among women worldwide. RNA-binding proteins (RBPs) are promising novel biomarkers for patients with malignant tumors, the abnormal expression of which is closely associated with the development of breast cancer. This study aimed to identify RBPs associated with the survival outcomes of breast cancer and to construct a prognostic model and a clinical prediction nomogram for breast cancer.

\section{Methods}

The transcriptome data of breast cancer were downloaded from TCGA database. GO and KEGG analyses were performed to clarify the gene functions and regulatory pathway. Cox and LASSO regression analyses were utilized to analyze the prognosis prediction effect of RBPs and clinical characteristics in breast cancer and create a risk score model. A nomogram was also built by merging the model and clinicaopatholigical characteristics, which was validated using calibration curves.

\section{Results}

A prognostic risk score model of the 22-RBP signature was established. This risk score predicted 3-, 5-, and 10-year overall survival rates sensitively and accurately. Compared to other clinicaopatholigical characteristics, this risk score had better predictive ability. We also constructed a nomogram based on risk scores and clinicaopatholigical characteristics. The nomogram may predict the 1-, 3-, and 5-year survival rates of patients with breast cancer.

\section{Conclusions}

RBPs play an important role in the development and survival outcomes of breast cancer by regulating multiple biological processes. Furthermore, this study identified and constructed a 22-RBP based signature and a clinical nomogram for predicting the survival probability of patients with breast cancer.

\section{Background}

In 2020, the global cancer data showed that breast cancer (BC) had exceeded lung cancer to become the most frequently diagnosed cancer in women. In 2020, there were 2.26 million newly diagnosed BC, representing $24.5 \%$ of all new cancer cases in women, with more than 680,000 deaths, accounting for $15.5 \%$ of cancer deaths (1). High incidence and mortality are important factors affecting the survival time of women with BC. 
Post-transcriptional gene regulation (PTGR) is an important biological process (2). It can maintain cell metabolism and coordinate the maturation, transport, stabilization, and degradation of many types of RNA. RNA-binding proteins (RBPs) are a class of highly conserved RNA-interacting proteins. About $60 \%$ of the 1,542 discovered RBPs are expressed in tissue-specific ways. These proteins can bind to multiple RNA (including rRNA, ncRNAs, snRNAs, and so on) to produce nuclear RNA complexes to perform their functions and have been key post-transcriptional regulators (3). They are involved in almost all procedures of PTGR, which can determine the fate and function of everytranscript in the cancer cell (4). Evidence suggests that RBP plays a critical role in regulating physiological balance, cell development, and stress response (5). RBP imbalance can lead to changes in RNA metabolism, resulting in changes in the cell transcriptome and proteome, thereby affecting cell growth, proliferation, and invasion (6). In addition, there is increasing evidence suggesting that RBP disorders are associated with tumor progression and prognosis. HuR has been identified as an RBP that acts as an important oncogenic driver that controls mRNA stability and promotes proliferation and metastasis of gastric cancer (7). RBP MEX3A can independently reflect the clinical course of $B C$, and MEX3A overexpression predicts poor prognosis (8-9). Although these findings are informative, to date, few researches have systematically evaluated the prognostic value of RBP in BC.

In this study, we performed an comprehensive analysis based on the data gained from The Cancer Genome Atlas (TCGA) database. We used Cox regression and survival analyses to establish prognostic RBP-related BC gene signatures. We also built a prognostic nomogram based on risk score and clinicopathological features to predict the survival outcomes of BC.

\section{Methods}

Data acquisition and RNA-binding protein screening

From the TCGA dataset (https://portal.gdc.com), the raw count of tumor RNA sequencing data (level 3) and the clinical information were obtained using the TCGAbiolinks package (version 2.20.0) (10). We obtained human RBP data from this study (3). Survival analyses were used to explore the correlations between the key prognostic genes and prognoses. In addition, we also generated Venn diagrams by the Draw Venn Diagram tool to identify key prognostic RBPs.

RBP signature establishment and verification

Hub RBPs were identified, and the predictive signature was constructed using the least absolute shrinkage and selection operator (LASSO) Cox regression and multivariate Cox analysis. The LASSO regression analysis can help narrow down the candidate gene number further. Cox proportional hazards models were utilized to estimate the hazard ratios (HRs) and to calculate the coefficients for risk RBPs in the equations. Survival analysis and time-dependent receiver operating characteristic (ROC) analyses were utilied to validate the model. 
Gene Ontology (GO) functional and Kyoto Encyclopedia of Genes and Genomes (KEGG) pathway enrichment analysis

GO and KEGG pathway analysis were performed to explore the biological functions and pathways of the differentially expressed RBPs.GO analysis were constituted by biological process (BP), cellular component (CC), and molecular function (MF). All these analyses were conducted by clusterProfiler (11), ggplot2, and GOplot (12) functions to present the results (12).

Protein-protein interaction (PPI) network construction

The STRING database (http://string-db.org) was utilized to build PPI network of the differentially expressed RBPs. Then Cytoscape 3.8.2 (http://cytoscape.org/) software was used to analyze the data gained from the STRING database. Furthermore, Molecular Complex Detection, was used to screen hub RBPs of the network.

Statistical analyses

In the present study, univariate and multivariate Cox regression, LASSO regression, Kaplan-Meier curveand log-rank test were applied. For the Kaplan-Meier curve, the p-value and HR were obtained using the log-rank test and univariate Cox regression. Furthermore, the nomogram and calibration curves were drawn by the "rms" package. LASSO regression was performed using the "glmnet" package. Forest plots were constructed using the geom_violin (ggplot2). All these analysis methods and R software packages were implemented using $R$ version 4.1.1 software (13). $P<0.05$ was considered as statistically significant.

\section{Results}

\section{Data analysis}

RNA-Seq and clinical data of 1,097 BC and 113 normal cases were gained from the TCGA database. The baseline for the inclusion of clinical data only for female patients was presented in Table 1. We further performed survival analysis of the whole genome of $\mathrm{BC}$, using univariate Cox and log-rank methods, according to the standard of $p<0.05$, finally obtaining 973 genes with significant prognosis (Additional file 1). Seventy-five RBPs with significant prognostic significance were finally obtained from the 1,542 RBPs included in the research. High and low risks were defined as HRs $>1$ and $<1$, respectively. Finally, there were 28 low-risk and 47 high-risk RBPs. HR values with 95\% Cls for the top 20 significantly prognostic RBPs are shown in the forest plot (Figure 1). 
Table 1

The baseline table for the inclusion of clinical data only for female patients

\begin{tabular}{|c|c|c|}
\hline Variables & Number of patients & $\%$ \\
\hline \multicolumn{3}{|l|}{ Age } \\
\hline Mean(SD) & $58.3(13.2)$ & \\
\hline Median [MIN, MAX] & $58[26,90]$ & \\
\hline \multicolumn{3}{|l|}{ Race } \\
\hline WHITE & 751 & $69.2 \%$ \\
\hline BLACK & 179 & $16.5 \%$ \\
\hline ASIAN & 61 & $5.6 \%$ \\
\hline AMERICAN INDIAN & 1 & $0.1 \%$ \\
\hline \multicolumn{3}{|l|}{ T stage } \\
\hline T1 & 279 & $25.7 \%$ \\
\hline T2 & 627 & $57.8 \%$ \\
\hline T3 & 137 & $12.6 \%$ \\
\hline T4 & 39 & $3.6 \%$ \\
\hline TX & 3 & $0.3 \%$ \\
\hline \multicolumn{3}{|l|}{$\mathrm{N}$ stage } \\
\hline NO & 510 & $47.0 \%$ \\
\hline N1 & 359 & $33.1 \%$ \\
\hline N2 & 119 & $11.0 \%$ \\
\hline N3 & 77 & $7.1 \%$ \\
\hline NX & 20 & $1.8 \%$ \\
\hline \multicolumn{3}{|l|}{ M stage } \\
\hline MO & 897 & $82.7 \%$ \\
\hline M1 & 21 & $1.9 \%$ \\
\hline$M X$ & 161 & $14.8 \%$ \\
\hline \multicolumn{3}{|l|}{ TNM stage } \\
\hline I & 181 & $16.7 \%$ \\
\hline
\end{tabular}




\begin{tabular}{|lll|}
\hline Variables & Number of patients & $\%$ \\
\hline II & 616 & $56.8 \%$ \\
\hline III & 246 & $22.7 \%$ \\
IV & 19 & $1.8 \%$ \\
\hline X & 12 & $1.1 \%$ \\
\hline Status & & \\
Alive & 932 & $85.9 \%$ \\
\hline Dead & 153 & $14.1 \%$ \\
\hline
\end{tabular}

Enrichment analysis results of significantly prognostic RBPs

To further understand the potential molecular mechanisms and functions of these RBPs, we performed GO and KEGG pathway analyses for all these significantly prognostic RBPs. Regarding BPs, low-risk RBPs were mainly enriched in cotranslational protein targeting to membrane, signal recognition particledependent cotranslational protein targeting to membrane, and so on. High-risk RBPs were mostly enriched in ncRNA processing, translational termination, ribosome biogenesis, mitochondrial translational termination, and protein-containing complex disassembly. Regarding CCs, low-risk RBPs were mainly enriched in cytosolic ribosomes, ribosomal subunits, cytosolic parts, ribosomes, and cytosolic large ribosomal subunits. However, the high-risk RBPs were notably enriched in ribosomes, ribosomal subunits, organellar ribosomes, mitochondrial ribosomes, and small ribosomal subunits. In the MF category, lowrisk RBPs were associated with structural constituents of ribosomes, rRNA binding, cytidine deaminase activity, translation elongation factor activity, and deaminase activity. However, the high-risk RBPs were enriched in RNA 7-methylguanosine cap binding, rRNA binding, and so on. KEGG pathway analysis presented that low-risk RBPs were also mainly related to spliceosomes and ribosomes, whereas high-risk RBPs were also mainly related to ribosomes, ribosome biogenesis in eukaryotes, and RNA transport (Figure 2).

Results of PPI network and screening hub RBPs

We further explored the effects of prognosis-related RBPs in BC by constructing a PPI network (Figure $3 A$ ). Furthermore, we analyzed the network to identify hub gene by the MODE plugin and identified a hub gene module with 25 RBPs (Figure 3B).

Establishment and verification of the risk core signature

Based on the 75 prognosis-associated RBPs, we performed LASSO regression to identify RBPs which have the highest potential prognostic effect. Eveuntually, 22 hub RBPs were obtained and utilized to construct a predictive signature (Figure 4A-B). Among these 22 RBPs, the 10 low-risk genes were CPSF4, RPL 14, EEF1B2, HNRNPA1, RPL38, PTRHD1, ENDOV, PARP12, APOBEC3F, and EIF5AL1; the 12 high-risk 
genes were MCTS1, MRPS18C, AIMP2, MRPL18, ZMAT3, LARP1, MRPS2, AIMP1, POP1, IGF2BP1, MRPS28, and NANOS1. We then applied the expression levels of the 22 hub RBPs and the regression coefficients to calculate the risk score for every patient. Risk scores were calculated by the equation: risk score $=(0.0845) \times$ MRPS $28+(0.0582) \times$ MRPS18C $+(-0.008) \times$ APOBEC3F $+(-0.2642) \times$ PARP12 + $(0.1008) \times \mathrm{POP} 1+(-0.1313) \times \mathrm{RPL} 38+(-0.1801) \times \mathrm{RPL} 14+(-0.1237) \times \mathrm{HNRNPA} 1+(-0.0218) \times \mathrm{PTRHD} 1$ $+(-0.2469) \times \mathrm{ENDOV}+(0.046) \times \mathrm{LARP} 1+(-0.1971) \times \mathrm{EEF} 1 \mathrm{~B} 2+(0.0046) \times \mathrm{AIMP} 2+(-0.033) \times \mathrm{EIF} 5 \mathrm{AL} 1+$ $(0.3038) \times \mathrm{ZMAT} 3+(0.4818) \times \mathrm{MCTS} 1+(0.3771) \times \mathrm{MRPL} 18+(0.0231) \times \mathrm{AIMP} 1+(0.5333) \times \mathrm{MRPS} 2+$ $(-0.1478) \times$ CPSF $4+(0.0517) \times$ NANOS1 $+(0.1103) \times$ IGF2BP1. Data from patients with BC were dichotomized into the high- and low-risk groups using the median risk score as the cutoff value. Patients in high-risk group have significantly shorter overall survival (OS) than patients in the low-risk group (Figure 4C). Furthermore, the 3-, 5-, and 10-year areas under the curve (AUCs) of the ROC curve generated to evaluate the prognostic ability of the signature were $0.794,0.767$, and 0.811 , respectively, indicating that the 22-RBP model had moderate diagnostic performance (Figure 4D). Next, we ranked patients with $\mathrm{BC}$ according to their risk scores and analyzed the survival status of every patient on a dot plot. The results showed that patients in the low-risk group have higher survival rates and times than those patients in the high-risk group.

The expression levels of the 22 hub RBPs in the high- and low-risk groups were also shown in Figure 4E.

Relationships between risk score and clinicopathological characteristics

Univariate and multivariate Cox regression analyses of TCGA data were conducted to determine whether the risk score was an independent prognostic predictors. Univariate analysis showed that $T, N, M$, and tumor-node-metastasis (TNM) stages and risk scores were significantly associated with OS in patients with BC. Furthermore, multivariate analysis predicted that $T, N, M$, and $T N M$ stages and risk scores were independent prognostic predictors for OS (Table 2). In conclusion, we can say that the risk score based on 22 RBPs may serve as an independent prognostic predictor for survival in patients with BC. To further explore the prognostic effect of the signature, we analyzed the association between the risk score and clinicopathological parameters. The results suggested that the risk score was significantly related to the $\mathrm{M}$ stage; the greater the grade of metastasis, the higher the risk score. However, risk scores were not related to the $T$ stage, $N$ stageand $T N M$ stage (Figure 5). The above results further confirmed that the risk score could be an independent prognostic predictor in patients with BC.

Table 2 Univariate and multivariate Cox regression analyses of TCGA data 


\begin{tabular}{|c|c|c|c|c|c|c|}
\hline & \multicolumn{3}{|c|}{ Univariable analysis } & \multicolumn{3}{|c|}{ Multivariable analysis } \\
\hline & $H R$ & $95 \% \mathrm{Cl}$ & P Value & $\mathrm{HR}$ & $95 \% \mathrm{Cl}$ & P Value \\
\hline RiskScore & 4.119 & $3.115-5.445$ & $<0.001 * \star \star$ & 3.335 & $2.516-4.421$ & $<0.001 \star \star \star \star$ \\
\hline \multicolumn{7}{|l|}{ T stage } \\
\hline T1 & reference & & & reference & & \\
\hline T2 & 0.037 & $0.010-0.142$ & $<0.001 * \star \star$ & 0.091 & $0.020-0.411$ & 0.002 ** \\
\hline T3 & 0 & $0.000-0.003$ & $<0.001 * \star \star$ & 0.001 & $0.000-0.012$ & $<0.001 * \star \star$ \\
\hline T4 & 0 & $0.000-0.003$ & $<0.001 * \star \star$ & 0.001 & $0.000-0.012$ & $<0.001 * \star \star$ \\
\hline \multicolumn{7}{|l|}{$\mathrm{N}$ stage } \\
\hline NO & reference & & & reference & & \\
\hline N1 & 0 & $0.000-\operatorname{Inf}$ & 0.998 & 0 & $0.000-\operatorname{lnf}$ & 0.997 \\
\hline N2 & 0 & $0.000-\operatorname{lnf}$ & 0.996 & 0 & $0.000-\operatorname{lnf}$ & 0.995 \\
\hline N3 & 0 & $0.000-\operatorname{lnf}$ & 0.994 & 0 & $0.000-\operatorname{lnf}$ & 0.994 \\
\hline \multicolumn{7}{|l|}{ M stage } \\
\hline MO & reference & & & reference & & \\
\hline M1 & 3.508 & $1.974-6.232$ & $<0.001 * \star \star$ & 4.099 & $2.189-7.676$ & $<0.001 * \star \star$ \\
\hline \multicolumn{7}{|l|}{ TNM stage } \\
\hline Stage1 & reference & & & reference & & \\
\hline Stage2 & 0.142 & $0.056-0.359$ & $<0.001 * \star *$ & 1.242 & $0.450-3.426$ & 0.675 \\
\hline Stage3 & 0.032 & $0.012-0.089$ & $<0.001$ *** & 0.227 & $0.068-0.760$ & 0.016 * \\
\hline Stage4 & 0.036 & $0.009-0.136$ & $<0.001 * \star \star$ & 0.142 & $0.026-0.772$ & 0.024 * \\
\hline
\end{tabular}

Establishment and verification of prediction nomogram

To develop a quantitative method for predicting the survival outcomes of patients with $\mathrm{BC}$, we built a nomogram that integrated risk score and $T$ stage, $N$ stage, and $M$ stage. Every variable was assigned a score; the scores of the four variables were then added, and a vertical line was drawn from the total score to the nomogram point scale to determine the estimated 1-, 3-, and 5-year survival rates (Figure 6A). The C-index value of the nomogram was 0.917 (0.908-0.927) for this BC cohort, suggesting that this nomogram had good discrimination capability. The calibration curves suggested that the nomogram 
were significantly consistent with actual observations for 1-, 3-, and 5-year OS in the TCGA-BC cohort, indicating that it was trustable (Figure 6B-D).

\section{Discussion}

Breast cancer has the highest incidence and mortality rates among women worldwide (1).

Clinicopathologicalfeatures, such as age, clinical stage, and tumor classification are major predictors of survival outcomes in patients with BC (14). Recent researches have shown that RBP is closely related to the occurrence and development of various cancers and plays a critical role in tumor invasion, migration, and metastasis (15). However, only a few RBP mechanisms have been studied, and the role of RBP in BC is not fully understood. Therefore, compared with a single clinicopathological parameter, establishing genetic markers and a nomogram using multiple biomarkers and clinicopathological features is a relatively effective method for predicting tumor prognosis. Therefore, signatures and nomograms based on RBPs may be more accurate in predicting BC survival.

In this research, we used RNA-Seq data and clinical data from the TCGA database for BC and identified 75 RBPs with significant prognostic significance. The biological functions and pathways of significant prognostic RBPs in BC were investigated via PPI network analysis and functional enrichment analysis. Next, we used LASSO and Cox regression analysis to screen out 22 key RBPs (CPSF4, RPL14, EEF1B2, HNRNPA1, RPL38, PTRHD1, ENDOV, PARP12, APOBEC3F, EIF5AL1, MCTS1, MRPS18C, AIMP2, MRPL18, ZMAT3, LARP1, MRPS2, AIMP1, POP1, IGF2BP1, MRPS28, NANOS1) most associated with prognosis. Based on 22 RBPs associated with prognosis, we established a promising 22-RBP signature and nomogram to predict the survival outcome of patients with BC. Based on our risk score signature, survival analysis showed significant differences in OS between the high- and low-risk subgroups, with the low-risk group generally surviving better than the high-risk group. The ROC curve showed that our prognostic model had good accuracy, with AUC values greater than 0.75 at 3, 5, and 10 years. Some studies have presented that stage is an independent prognostic indicator of $\mathrm{BC}$, and the risk score also has good predictive performance, even equal to the predictive ability of the TNM stage.

We divided the 22 RBPs with significant survival significance into high-risk and low-risk RBPs, according to the HR value of survival analysis. The high-risk RBPs were MCTS1, MRPS18C, AIMP2, MRPL18, ZMAT3, LARP1, MRPS2, AIMP1, POP1, IGF2BP1, MRPS28, and NANOS1. Low-risk RBPs were CPSF4, RPL14, EEF1B2, HNRNPA1, RPL38, PTRHD1, ENDOV, PARP12, APOBEC3F, and EIF5AL1. Poly (ADP-ribose) polymerase 12 (PARP12) is a mono-ADP-ribosyltransferase. Studies have shown that PARP12 deficiency promotes the migration and invasion of hepatocellular carcinoma (HCC) cells and increases HCC metastasis. This is similar to the conclusion of this study that PARP12 is a protective RBP in BC (16). Cleavage and polyadenylation specificity factor 4 (CPSF4) is a member of the CPSF complex, suggesting that it has low expression and poor survival in breast tumor tissues. In contrast to Lee's study (17), CPSF4 was highly expressed in triple-negative $\mathrm{BC}$ and promoted the invasion and migration of $\mathrm{BC}$ cells. This may be due to the high expression of CPSF4 in certain tumor phenotypes, such as lung and liver cancers $(18,19)$. Studies $(20)$ have shown that RPL14, associated with various tumors, has lower expression in 
various tumors and inhibits metastasis, invasion, and epithelial-to-mesenchymal transition. In this study, as a protective RBP, the high expression of RPL14 can significantly increase the survival time of patients, which is consistent with the finding of Lin's study. RPL38 belongs to the L38E family of ribosomal proteins and is located in the cytoplasm of cancer cells. A previous study (21) showed that knockdown of RPL38 decreased the proliferation and invasion of gastric cancer cells. In this study, we observed a trend of low expression in breast tumor tissues. As an oncogene, MCTS1 canpromote tumor cell proliferation and migration and inhibit apoptosis. This further indicates that MCTS1 is a high-risk gene in this study (22-24). Studies have found that AIMP2 plays a key role in the control of cell fate. It showed antiproliferative activity by enhancing the growth arrest signal of tumor growth factor- $\beta$ signaling. AIMP2 also promotes cell death by activating p53 and tumor necrosis factor-a apoptotic signals (25). However, a splicing variant of AIMP2, which disrupts the normal function of AIMP2 and induces tumorigenesis, is more common in tumor tissues (26). This further indicates that the patient was classified as a high-risk RBP. ZMAT3 is an RNA splicing and homeostasis regulator. As an important downstream splicing regulator of p53 (27), ZMAT3 plays a vital role in p53-mediated tumor suppression (28). In the LARP1 interaction group, mRNA transcription of mTOR was stabilized by LARP1. At the functional level, LARP1 can promote cell migration, invasion, unanchored growth, and tumorigenesis in vivo (29). In addition, LARP1 expression increased in epithelial cancers such as cervical cancer (30), whose expression is associated with disease progression and poor prognosis, respectively. LARP1 may be a promising therapeutic target. Studies have shown that IGF2BP is a novel oncogenic factor in many solid tumors, including ovarian cancer and breast cancer, and its high expression is closely related to metastasis and poor prognosis (31). Current studies have suggested that IGF2BP1 can be used as a therapeutic target and may be used in the clinical diagnosis and prognostic evaluation of bladder cancer and neuroblastoma (32-33).

At present, a prognostic model based on BC prognostic-associated RBP has not been reported. This is the first study to use multiple prognostic-associated RBP genes to built a prognostic model for patients with BC. Although our model has good predictive performance, some limitations also need to be discussed. First, 22 RBP gene signatures were constructed based on the TCGA-BCRA dataset; however, they could not be verified in clinical practice and the external cohort. Second, the races of patients with $B C$ in the TCGA database are white and black, and it is not clear whether the predictive effect is the same among Asians and other races. In addition, whether this clinical prediction model has good validity in other BC subtypes requires further verification. Finally, our study is retrospective and needs to be further validated in larger prospective studies.

\section{Conclusions}

In this study, We found RBPs played an important role in the development and survival outcomes of BC by regulating multiple biological processes. Furthermore, we identified and constructed a 22-RBP based signature and a clinical nomogram for predicting the survival of patients. We think our results may assist in prognosis management postoperative intervention of high-risk patients with BC clinically. 


\section{Abbreviations}

$\mathrm{BC}$ breast cancer

BP biological process

CC cellular component

GO Gene Ontology

HRs hazard ratios

KEGG Kyoto Encyclopedia of Genes and Genomes

LASSO Least absolute shrinkage and selection operator

MF molecular function

PPI Protein-protein interaction

PTGR Post-transcriptional gene regulation

RBPs RNA-binding proteins

ROC receiver operating characteristic

TCGA The Cancer Genome Atlas

TNM tumor-node-metastasis

\section{Declarations}

\section{Ethics approval and consent to participate}

Not applicable

Consent for publication

Not applicable

\section{Availability of data and materials}

These data and materials can be available from the corresponding author for rational reasons.

\section{Competing interests}

The authors declare that they have no competing interests 


\section{Funding}

This research was supported by Natural Science Foundation of Liaoning Province (2020-MS-178).

\section{Authors' contributions}

Xi Gu and Xudong Zhu designed this research. Tong Zhu and Jiawen Bu analyzed these data from public databases. Tongzhu, Xudong Zhu, Jiawen Bu, Yi Jiang, JinqiXue, and Nan Niuwrote this paper. All authors have read and approved the final manuscript.

\section{Acknowledgments}

Not applicable

\section{References}

1. Sung H, Ferlay J, Siegel RL, Laversanne M, Soerjomataram I, Jemal A, Bray F. Global Cancer Statistics 2020: GLOBOCAN Estimates of Incidence and Mortality Worldwide for 36 Cancers in 185 Countries. CA Cancer J Clin. 2021; 71:209-49.

2. Gehring NH, Wahle E, Fischer U. Deciphering the mRNP Code: RNA-Bound Determinants of PostTranscriptional Gene Regulation. Trends Biochem Sci. 2017;42:369-82.

3. Gerstberger S, Hafner M, Tuschl T. A census of human RNA-binding proteins. Nat Rev Genet. 2014;15:829-45.

4. Pereira B, Billaud M, Almeida R. RNA-Binding Proteins in Cancer: Old Players and New Actors. Trends Cancer. 2017;3:506-28.

5. Masuda K, Kuwano Y. Diverse roles of RNA-binding proteins in cancer traits and their implications in gastrointestinal cancers. Wiley Interdiscip Rev RNA. 2019;10:e1520.

6. Mohibi S, Chen X, Zhang J. Cancer the'RBP'eutics-RNA-binding proteins as therapeutic targets for cancer. PharmacolTher. $2019 ; 203: 107390$.

7. Yang F, Hu A, Li D, Wang J, Guo Y, Liu Y, et al. Circ-HuR suppresses HuR expression and gastric cancer progression by inhibiting CNBP transactivation. Mol Cancer. 2019;18:158.

8. Wang Y, Liang Q, Lei K, Zhu Q, Zeng D, Liu Y, et al. Targeting MEX3A attenuates metastasis of breast cancer via $\beta$-catenin signaling pathway inhibition. Cancer Lett. 2021;521:50-63.

9. Shi X, Sun Y, Zhang Y, Wang W, Xu J, Guan Y, et al. MEX3A promotes development and progression of breast cancer through regulation of PIK3CA. Exp Cell Res. 2021;404:112580.

10. Colaprico A, Silva TC, Olsen C, Garofano L, Cava C, GaroliniD,et al.TCGAbiolinks: an R/Bioconductor package for integrative analysis of TCGA data. Nucleic Acids Res. 2016;44:e71.

11. Yu G, Wang LG, Han Y, He QY. clusterProfiler: an R package for comparing biological themes among gene clusters. OMICS. 2012;16:284-7. 
12. Walter W, Sánchez-Cabo F, Ricote M. GOplot: an R package for visually combining expression data with functional analysis. Bioinformatics. 2015;31:2912-4.

13. Chan BKC. Data Analysis Using R Programming. Adv Exp Med Biol. 2018;1082:47-122.

14. Tokunaga E, ljichi $\mathrm{H}$, Tajiri W, Masuda T, Takizawa $\mathrm{K}$, Ueo $\mathrm{H}$, et al. The comparison of the anatomic stage and pathological prognostic stage according to the AJCC 8th edition for the prognosis in Japanese breast cancer patients: data from a single institution. Breast Cancer. 2020;27:1137-46.

15. Lujan DA, Ochoa JL, Hartley RS. Cold-inducible RNA binding protein in cancer and inflammation. Wiley Interdiscip Rev RNA. 2018;9:10.1002/wrna.1462.

16. Shao C, Qiu Y, Liu J, Feng H, Shen S, Saiyin H, et al. PARP12 (ARTD12) suppresses hepatocellular carcinoma metastasis through interacting with FHL2 and regulating its stability. Cell Death Dis. 2018;9:856.

17. Lee K, Zheng Q, Lu Q, Xu F, Qin G, Zhai Q, et al. CPSF4 promotes triple negative breast cancer metastasis by upregulating MDM4. Signal Transduct Target Ther. 2021;6:184.

18. Chen W, Qin L, Wang S, Li M, Shi D, Tian Y, et al. CPSF4 activates telomerase reverse transcriptase and predicts poor prognosis in human lung adenocarcinomas. Mol Oncol. 2014;8:704-16.

19. Wang X, Dong J, Li X, Cheng Z, Zhu Q. CPSF4 regulates circRNA formation and microRNA mediated gene silencing in hepatocellular carcinoma. Oncogene. 2021;40:4338-51.

20. Zhang Z, Zhang Y, Qiu Y, Mo W, Yang Z. Human/eukaryotic ribosomal protein L14 (RPL14/eL14) overexpression represses proliferation, migration, invasion and EMT process in nasopharyngeal carcinoma. Bioengineered. 2021;12:2175-86.

21. Ji H, Zhang X. RPL38 Regulates the Proliferation and Apoptosis of Gastric Cancer via miR-374b5p/VEGF Signal Pathway. Onco Targets Ther. 2020;13:6131-41.

22. Huang Z, Su Q, Li W, Ren H, Huang H, Wang A. MCTS1 promotes invasion and metastasis of oral cancer by modifying the EMT process. Ann Transl Med. 2021;9:997.

23. Gao C, Dong R, Li Y, Liang J, Tian H. MCTS1 promotes the development of lung adenocarcinoma by regulating E2F1 expression. Oncol Lett. 2021;22:531.

24. Tian C, Zeng S, Luo J. MCTS1 Directly Binds to TWF1 and Synergistically Modulate Cyclin D1 and CMyc Translation in Luminal A/B Breast Cancer Cells. Onco Targets Ther. 2020;13:5353-61.

25. Lim S, Cho HY, Kim DG, Roh Y, Son SY, Mushtaq AU, et al. Targeting the interaction of AIMP2-DX2 with HSP70 suppresses cancer development. Nat Chem Biol. 2020;16:31-41.

26. Ku J, Kim R, Kim D, Kim D, Song S, Lee K, et al. Single-cell analysis of AIMP2 splice variants informs on drug sensitivity and prognosis in hematologic cancer. Commun Biol. 2020;3:630.

27. Muys BR, Anastasakis DG, Claypool D, Pongor L, Li XL, Grammatikakisl,et al. The p53-induced RNAbinding protein ZMAT3 is a splicing regulator that inhibits the splicing of oncogenic CD44 variants in colorectal carcinoma. Genes Dev. 2021;35:102-16.

28. Bieging-Rolett KT, Kaiser AM, Morgens DW, Boutelle AM, Seoane JA, Van Nostrand EL, et al. Zmat3 Is a Key Splicing Regulator in the p53 Tumor Suppression Program. Mol Cell. 2020;80:452-69. 
29. Li M, Yin B, Chen M, Peng J, Mu X, Deng Z, et al. Downregulation of the IncRNA ASB16-AS1 Decreases LARP1 Expression and Promotes Clear Cell Renal Cell Carcinoma Progression via miR185-5p/miR-214-3p. Front Oncol. 2021;10:617105.

30. Mura M, Hopkins TG, Michael T, Abd-Latip N, Weir J, Aboagye E, et al. LARP1 post-transcriptionally regulates mTOR and contributes to cancer progression. Oncogene. 2015;34:5025-36.

31. Zhu S, Wang JZ, Chen D, He YT, Meng N, Chen M, et al. An oncopeptide regulates m6A recognition by the m6A reader IGF2BP1 and tumorigenesis. Nat Commun. 2020;11:1685.

32. Xie F, Huang C, Liu F, Zhang H, Xiao X, Sun J, et al. CircPTPRA blocks the recognition of RNA N6methyladenosine through interacting with IGF2BP1 to suppress bladder cancer progression. Mol Cancer. 2021;20:68.

33. Bell JL, Turlapati R, Liu T, Schulte JH, Hüttelmaier S. IGF2BP1 harbors prognostic significance by gene gain and diverse expression in neuroblastoma. J Clin Oncol. 2015;33:1285-93.

\section{Figures}


A

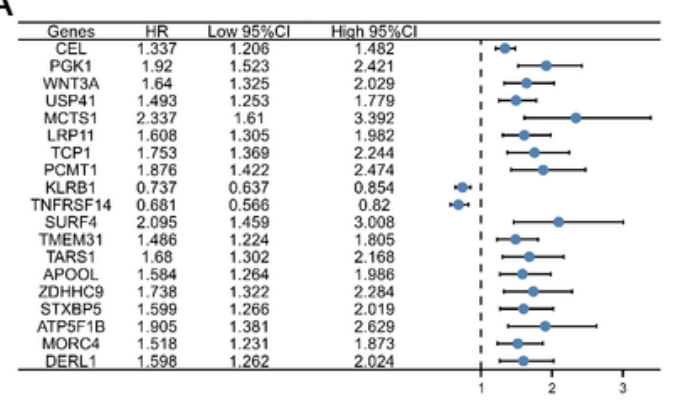

C

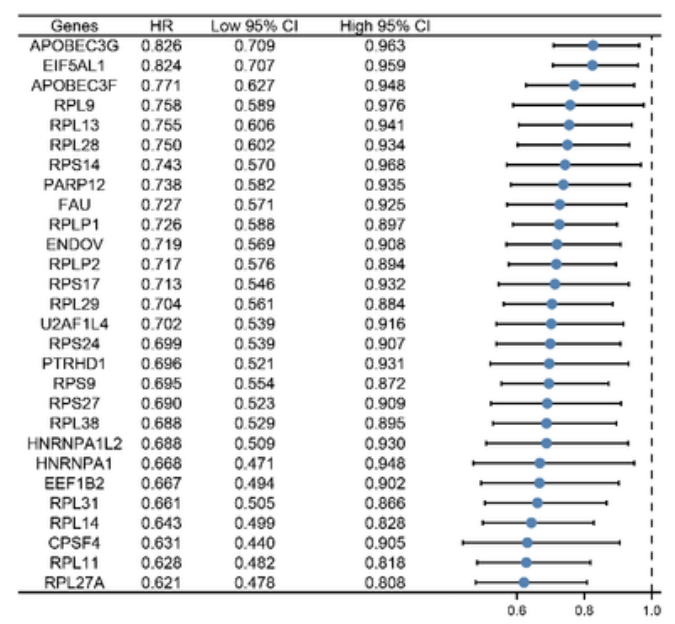

B

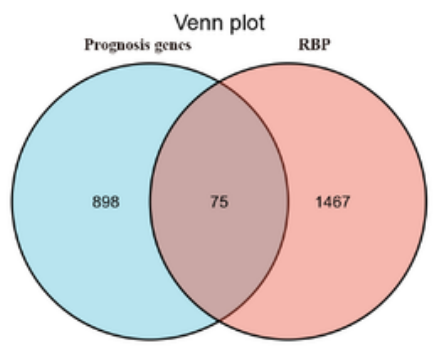

D

\begin{tabular}{|c|c|c|c|c|}
\hline $\begin{array}{c}\text { Geans } \\
\text { MCIS1 } \\
\text { MRPS18C }\end{array}$ & & & 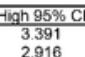 & \\
\hline $\begin{array}{l}\text { MRPS18C } \\
\text { PTGES3 } 3\end{array}$ & 1.881 1.855 & $\begin{array}{l}\frac{1.213}{1.338} \\
1.30\end{array}$ & $\begin{array}{l}2.216 \\
2.573\end{array}$ & $\Longrightarrow$ \\
\hline $\begin{array}{l}\text { NoNO } \\
\text { PNO1 }\end{array}$ & 1. 1.675 & $\begin{array}{l}\text { 1).1999 } \\
1.207\end{array}$ & $\begin{array}{l}2.477 \\
2.324\end{array}$ & $=$ \\
\hline 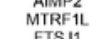 & . 6540 & $\begin{array}{l}1.1900 \\
1.171\end{array}$ & $\begin{array}{l}2.310 \\
2296\end{array}$ & $\Rightarrow$ \\
\hline MRPL18 & 1.564 & 1.165 & $\begin{array}{l}2.153 \\
2.153 \\
2\end{array}$ & $=$ \\
\hline 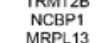 & . 1.533 & $\begin{array}{l}1.1665 \\
1.155\end{array}$ & $\begin{array}{l}2.033 \\
2.034 \\
\end{array}$ & $=$ \\
\hline & .1.529 & $\begin{array}{l}1.233 \\
1.147 \\
\end{array}$ & $\begin{array}{l}1.987 \\
2.031\end{array}$ & \\
\hline $\begin{array}{l}\text { LARP1 } \\
\text { ARPS2 }\end{array}$ & $\begin{array}{l}4.489 \\
4.481 \\
478\end{array}$ & $1: 191$ & $\begin{array}{l}1.9863 \\
1.983\end{array}$ & $\sigma^{\infty}$ \\
\hline $\begin{array}{l}\text { FAPSB } \\
\text { AMMP }\end{array}$ & $\begin{array}{l}4.468 \\
4.465 \\
4.65\end{array}$ & 1.059 & $\begin{array}{l}2.03935 \\
2.035\end{array}$ & 吅 \\
\hline $\begin{array}{l}\text { STRAP } \\
\text { SOPP }\end{array}$ & $\begin{array}{l}\text {. } 1445 \\
\text { 1.414 }\end{array}$ & $\begin{array}{l}1.094 \\
1.1099 \\
1.195\end{array}$ & $\begin{array}{l}1.900 \\
1.900 \\
1.759\end{array}$ & $=$ \\
\hline $\begin{array}{l}\text { MPHOSPH } 10 \\
\text { UTP2 } 33\end{array}$ & $\begin{array}{l}4.42 \\
4355\end{array}$ & $\begin{array}{l}1.1017 \\
1.174\end{array}$ & $\begin{array}{l}2043 \\
1.774\end{array}$ & $=$ \\
\hline $\begin{array}{l}\text { RSRC1 } \\
\text { HINT3 }\end{array}$ & $\begin{array}{l}1.440 \\
1.419\end{array}$ & $\begin{array}{l}1.030 \\
1.127\end{array}$ & $\begin{array}{l}1.967 \\
1.787\end{array}$ & $=$ \\
\hline 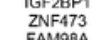 & 1.1399 & 1.052 & $\begin{array}{l}1.884 \\
1.861 \\
.1960\end{array}$ & 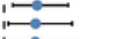 \\
\hline 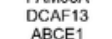 & $\begin{array}{l}1.383 \\
.377 \\
.377\end{array}$ & $\begin{array}{l}1.125 \\
1.1252 \\
1.052\end{array}$ & $\begin{array}{l}1.701 \\
1804\end{array}$ & $=-$ \\
\hline & 1.377 & 1.1.044 & 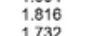 & \\
\hline 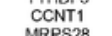 & . & $\begin{array}{l}1.052 \\
1.0692 \\
1069\end{array}$ & 1.782 & $=$ \\
\hline $\begin{array}{l}\text { EIF } 2 A 243 \\
\text { PABCP3 }\end{array}$ & . & $\begin{array}{l}1.096 \\
1017\end{array}$ & $\begin{array}{l}1.766 \\
1813\end{array}$ & 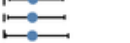 \\
\hline $\begin{array}{l}\text { DROSSA } \\
\text { XPOT }\end{array}$ & . 1354 & 1.021 & $\begin{array}{l}1.795 \\
1.670\end{array}$ & 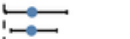 \\
\hline $\begin{array}{l}\text { RPF2 } \\
\text { CDC40 }\end{array}$ & .1.338 & $\begin{array}{l}1.039 \\
1.067\end{array}$ & $\begin{array}{l}1.723 \\
1.637 \\
\end{array}$ & $=$ \\
\hline $\mathrm{zCCHC14}$ & . 316 & 1.015 & 1.707 & $E_{0}^{\infty}$ \\
\hline 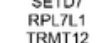 & 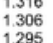 & $\begin{array}{l}1.0033 \\
1040 \\
1040\end{array}$ & 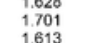 & 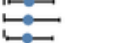 \\
\hline $\begin{array}{l}\text { STPPBP4 } \\
\text { NANOS1 }\end{array}$ & $\begin{array}{l}1.249 \\
1.246 \\
\end{array}$ & $\begin{array}{l}1.017 \\
1.098 \\
\end{array}$ & $\begin{array}{l}1.5444 \\
1.444\end{array}$ & $=$ \\
\hline
\end{tabular}

Figure 1

Screening for RNA-binding proteins (RBPs) in The Cancer Genome Atlas-BReast CAncer A: Forest plot of top 20 prognosis genes $B$ : Venn diagram of the prognosis genes and RBPs C: Forest plot of 28 low-risk RBPs D: Forest plot of 47 high-risk RBPs 
Figure 2
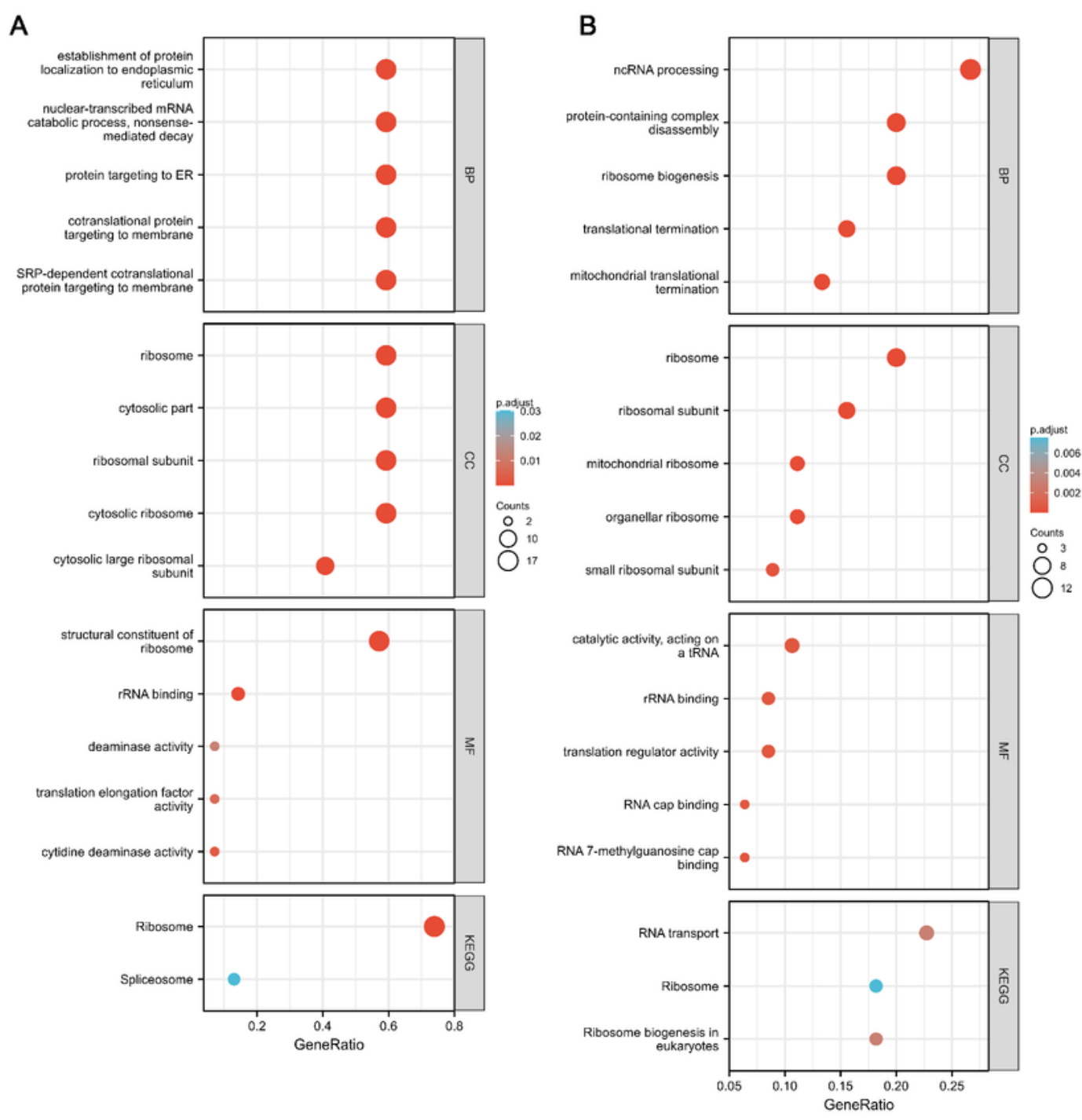

\section{Figure 2}

Functional enrichment analysis of the prognosis of RNA-binding proteins (RBPs) shown by bubble plots A: Gene Ontology (GO) and Kyoto Encyclopedia of Genes and Genomes (KEGG) enrichment analysis of 28 low-risk RBPs B: GO and KEGG enrichment analysis of 47 high-risk RBPs 
Figure 3

A

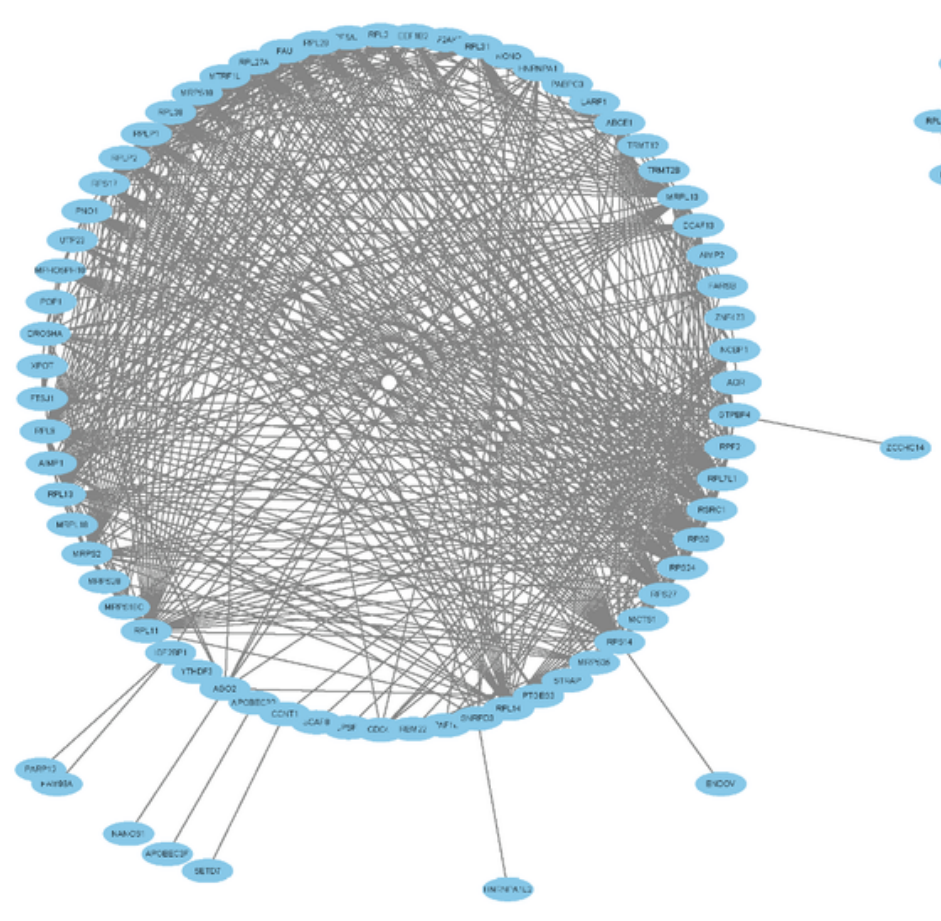

B

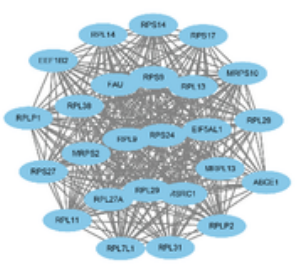

\section{Figure 3}

Network diagram of prognostic RNA-binding proteins (RBPs) A: Protein-protein interaction network of 75 prognostic RBPs B: 25 hub genes 
Figure 4

A

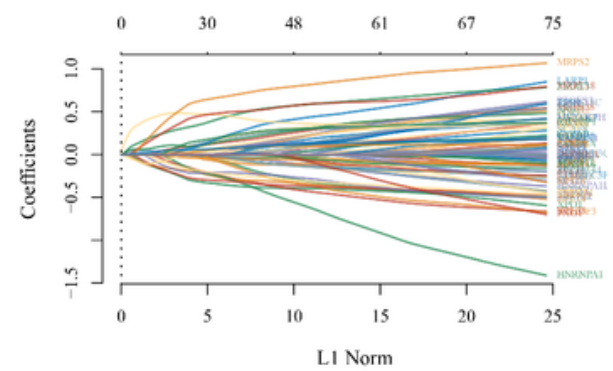

C

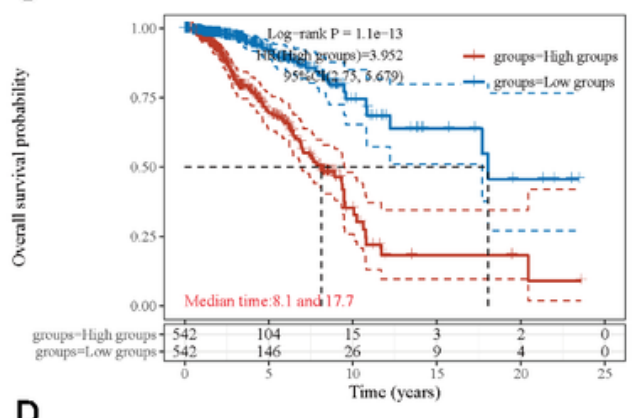

D

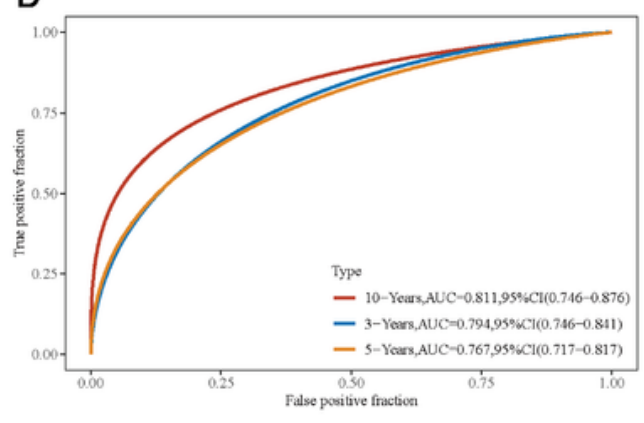

B

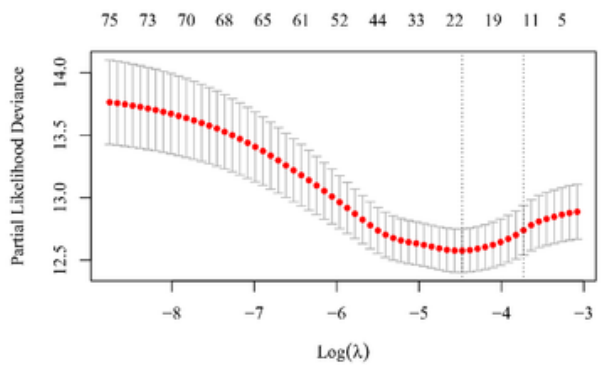

$E$

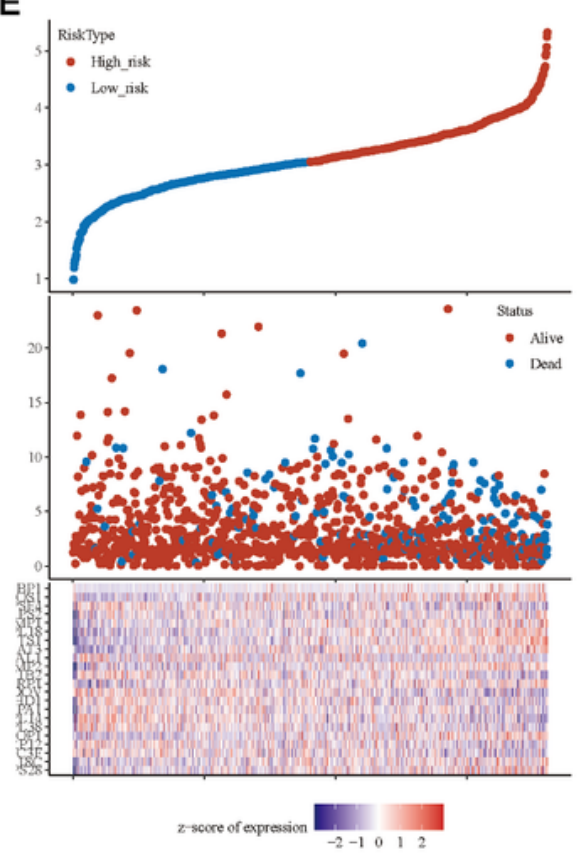

\section{Figure 4}

The LASSO regression analysis applied to screening prognostic RBPs and determination of risk scores by the prognostic model in the Stomach Adenocarcinoma dataset. A: LASSO coefficient profiles of 22 RBPs with nonzero coefficients determined by the optimal lambda B: Screening of the optimal parameter (lambda) at which vertical lines were drawn. C: Survival analysis of the high- and low-risk groups D: ROC 
curve analysis of the prognostic model E: Risk score distribution and survival status of patients with breast cancer in the Stomach Adenocarcinoma dataset.

Figure 5

A

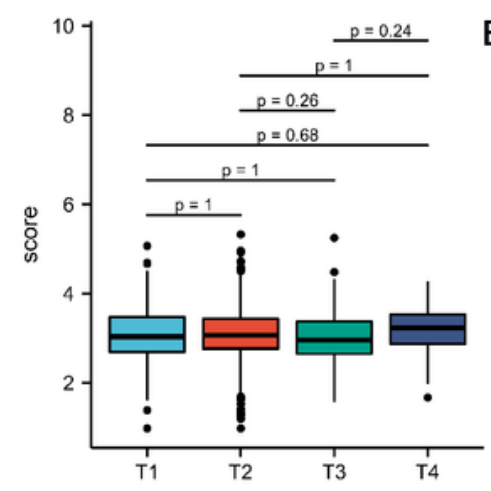

C

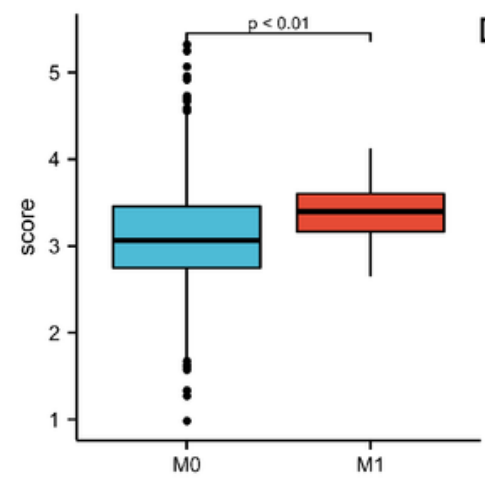

B

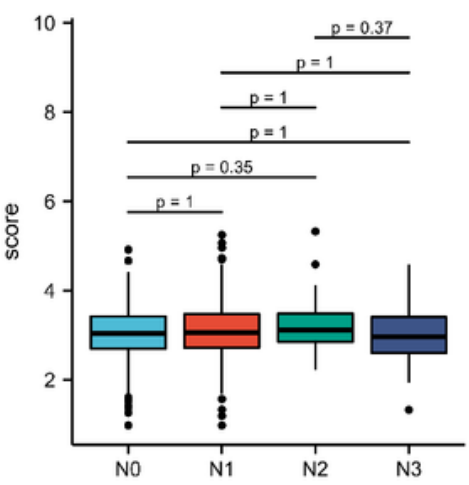

D

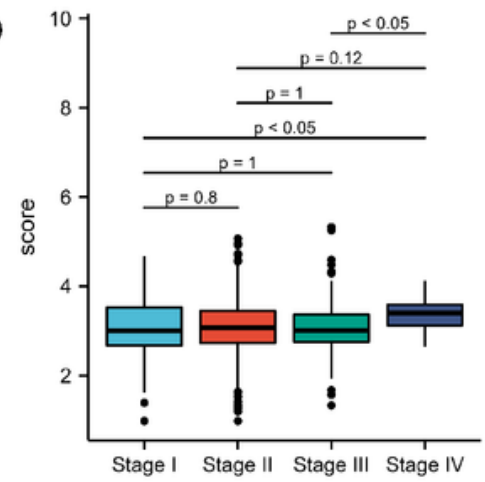

Figure 5

Prognostic risk scores correlated with clinicopathological features A-D: Distribution of risk scores stratified by $T(A), N(B), M(C)$, and TNM stages (D). 


\section{Figure 6}

A

Points

riskScore

Tumor

Node

Metastasis

Total Points

Linear Predictor

1-year Survival Probability

3-year Survival Probability

5-year Survival Probability
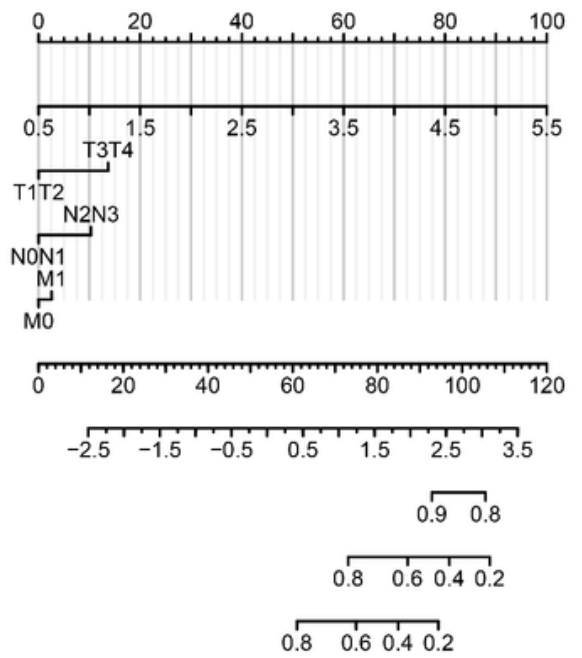

B

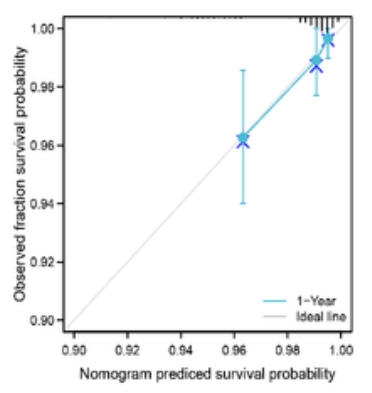

C

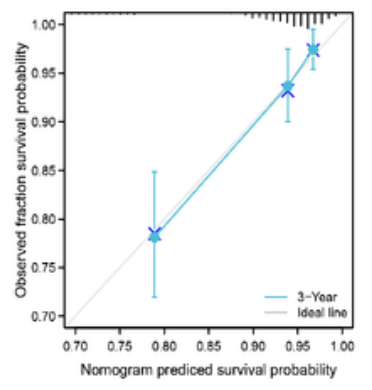

D

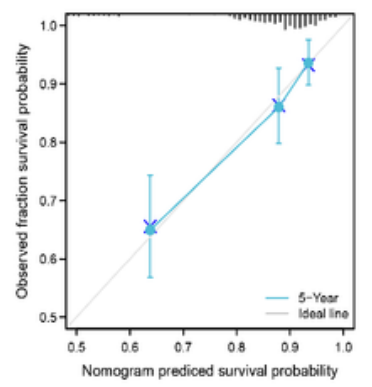

\section{Figure 6}

The nomogram for prediction on survival probability in patients with BC. A: Development of nomogram for predicting 1-, 3-, and 5-year overall survival in patients with BC B: The calibration curve for predicting the 1-year survival in patients with $\mathrm{BC}$ C: The calibration curve for predicting the 3-year survival in patients with $B C D$ : The calibration curve for predicting the 5-year survival in patients with $B C$ 


\section{Supplementary Files}

This is a list of supplementary files associated with this preprint. Click to download.

- Additionalfile1.xlsx 